\title{
Article \\ Flexible SAW Microfluidic Devices as Wearable pH Sensors Based on ZnO Nanoparticles
}

\author{
Luigi Piro ${ }^{1,2, *}$, Leonardo Lamanna ${ }^{3}$, Francesco Guido ${ }^{1}$, Antonio Balena $\left.{ }^{1,2}{ }^{(}\right)$, Massimo Mariello ${ }^{1,2}(\mathbb{D}$, \\ Francesco Rizzi ${ }^{1(1)}$ and Massimo De Vittorio ${ }^{1,2}$ () \\ 1 Center for Biomolecular Nanotechnologies, Istituto Italiano di Tecnologia, 73010 Arnesano, Italy; \\ francesco.guido@iit.it (F.G.); antonio.balena@iit.it (A.B.); massimo.mariello@iit.it (M.M.); \\ francesco.rizzi@iit.it (F.R.); massimo.devittorio@iit.it (M.D.V.) \\ 2 Department of Innovation Engineering, Campus Ecotekne, University of Salento, 73100 Lecce, Italy \\ 3 Center for Nano Science and Technology, Istituto Italiano di Tecnologia, 20133 Milan, Italy; \\ leonardo.lamanna@iit.it \\ * Correspondence: luigi.piro@iit.it
}

Citation: Piro, L.; Lamanna, L;

Guido, F.; Balena, A.; Mariello, M.; Rizzi, F; De Vittorio, M. Flexible SAW Microfluidic Devices as Wearable $\mathrm{pH}$ Sensors Based on $\mathrm{ZnO}$ Nanoparticles. Nanomaterials 2021, 11, 1479. https:// doi.org/10.3390/nano11061479

Academic Editor: Camelia Bala

Received: 30 April 2021

Accepted: 31 May 2021

Published: 3 June 2021

Publisher's Note: MDPI stays neutral with regard to jurisdictional claims in published maps and institutional affiliations.

Copyright: (c) 2021 by the authors. Licensee MDPI, Basel, Switzerland. This article is an open access article distributed under the terms and conditions of the Creative Commons Attribution (CC BY) license (https:// creativecommons.org/licenses/by/ $4.0 /)$.

\begin{abstract}
In this work, a new flexible and biocompatible microfluidic $\mathrm{pH}$ sensor based on surface acoustic waves (SAWs) is presented. The device consists of polyethylene naphthalate (PEN) as a flexible substrate on which aluminum nitride (AIN) has been deposited as a piezoelectric material. The fabrication of suitable interdigitated transducers (IDTs) generates Lamb waves (L-SAW) with a center frequency $\approx 500 \mathrm{MHz}$ traveling in the active region. A SU-8 microfluidics employing $\mathrm{ZnO}$ nanoparticles (NPs) functionalization as a $\mathrm{pH}$-sensitive layer is fabricated between the IDTs, causing a shift in the L-SAW resonance frequency as a function of the change in $\mathrm{pH}$ values. The obtained sensitivity of $\approx 30 \mathrm{kHz} / \mathrm{pH}$ from $\mathrm{pH} 7$ to $\mathrm{pH} 2$ demonstrates the high potential of flexible SAW devices to be used in the measurement of $\mathrm{pH}$ in fluids and biosensing.
\end{abstract}

Keywords: SAW; wearable device; flexible; biosensor; PEN; pH ZnO nanoparticles

\section{Introduction}

The development of biocompatible and flexible micro-electro-mechanical system (MEMS) sensors has increased over the last decade [1]. Surface acoustic wave (SAW)based devices represent one of the main building blocks in MEMS and have applications in many fields, mostly the development of biosensors for many different purposes $(\mathrm{pH}$, heavy metals concentrations) [2,3], and different physical (light intensity, temperature, deformation) [4-7] and biological (proteins, small molecule or cell concentrations) [8-10] parameters. Usually, SAW devices are fabricated on thick piezoelectric crystals as $\mathrm{LiNbO}_{3}$ or $\mathrm{LiTaO}_{3}$ [11]. These materials show excellent piezoelectric properties, but their high stiffness and expensive cost make them unsuitable for wearable applications. Recent progress in thin piezoelectric film deposition techniques has allowed thin AlN or $\mathrm{ZnO}$ to be deposited on film on soft substrates [1]. The demand for flexible and skin-compliant materials has increased in the last decade, and new flexible materials such as Kapton [12] or polyethylene naphthalate (PEN) [13] have been exploited. The introduction of such low-cost and flexible materials and the remote-control capability of SAW-based devices have made them good candidates for biosensors applications.

$\mathrm{pH}$ measurement is fundamental in many fields such as agriculture, chemistry, pharmaceuticals and biochemical processes, and, above all, its value is one of the most important parameters for monitoring physiological, biological and the medical state of human health [14]. Human fluids (sweat, saliva, blood, etc.) have their own physiological $\mathrm{pH}$ value range. If the $\mathrm{pH}$ values are detected out of the physiological range, this could be an efficient way to alert for illnesses and dysfunctions. For these reasons, many different $\mathrm{pH}$ sensors have been developed, based especially on electrochemical (potentiometric and 
conductometric) [15] and non-electrochemical (optical and calorimetric) [16] principles. Here, an innovative $\mathrm{pH}$ sensor based on SAWs is shown.

Polyethylene naphthalate (PEN), a thermoplastic polyester widely used in electronics, packaging, textile and industrial fibers, has been used as a substrate for SAW fabrication. PEN is a flexible, transparent and biocompatible material with good mechanical and electrical insulation properties [17]. It is also resistant to heat, moisture and many chemical solvents used in microfabrication processes [18]. Recently, aluminum nitride (AlN) has been used as a piezoelectric material in combination with PEN, which allows the generation and propagation of SAWs on the surface of the thin piezoelectric layer [19]. AlN has good piezoelectric and dielectric properties [20] and it is biocompatible [21]. In particular, c-axis (0002)-orientated AlN allows two principal wave propagation modes: Rayleigh waves (R-SAWs) and Lamb waves (L-SAWs). The R-SAWs are favored in the AlN that is grown on rigid substrates (usually silicon) and propagate at a phase velocity of about $5000 \mathrm{~m} / \mathrm{s}$, whereas the L-SAWs are favored in membranes and thin films and, when grown on flexible substrates, travel at a phase velocity of about $10000 \mathrm{~m} / \mathrm{s}$ [1]. Therefore, AlN presents a high SAW propagation velocity and a high breakdown voltage. There are several deposition techniques used to deposit AlN, such as laser ablation, chemical vapor deposition and reactive sputtering $[22,23]$. Thanks to recent progress, the last technique allows the deposition of good c-axis (0002)-orientated crystals of AlN at a low temperature [13]. These features make PEN/AIN a great combination for SAW devices. This new platform has been applied as an acoustic-based sensor for temperature, strain, light and E. coli cells' quantification [4,5,24]. The fundamental challenge is to integrate a microfluidic channel directly onto a SAW device in order to obtain a sensor that detects in real-time. The introduction of a microfluidic system on a SAW-based device and the functionalization of the microchannel active region allows the detection of many biochemical parameters in a liquid solution through SAW resonance frequency shift. The main issue in SAW-based lab-on-chip microfluidic devices is that when a liquid sample is in contact with IDTs it dampens the signal [25]. Hence, avoiding direct contact between the fluid sample and the transducers by introducing microfluidics, it has been possible to measure the S21 signal and its variation depending on the chemical or biological variations of the samples. $\mathrm{A} \approx 300 \mu \mathrm{m}$ thick microchannel has been fabricated directly on a SAW device and then functionalized with $\mathrm{ZnO}$ nanoparticles (NPs), which have become the most used metal oxide nanoparticles for biological applications due to their good biocompatibility, low toxicity and low cost. ZnO NPs have applicability in many fields including the rubber industry, the pharmaceutical and cosmetic industries, and the textile and electronic (photoelectronic, UV laser, solar cells, etc.) industries [26]. In this work, they are used as a $\mathrm{pH}$-sensitive layer due to their ability to change their conductivity depending on the $\mathrm{pH}$ value (in the acid region).

The working principle of an SAW device is based on the application of a radiofrequency (RF) signal to the metallic IDTs, which allows the generation and propagation of SAWs along the piezoelectric surface. The center frequency of these devices is given by Equation (1):

$$
\mathrm{f}_{0}=\mathrm{v} / \lambda
$$

where the velocity $\mathrm{v}$ depends on the substrate's nature and the type of the traveling wave, while the wavelength $\lambda$ depends on the period of the IDT.

The fundamental biosensing principle using acoustic waves is the measurement of the changes in the propagation velocity of the waves through the changes in the resonant frequency or phase angle. These variations can be due to extrinsic factors such as temperature, UV or IR light intensity, $\mathrm{pH}$, mass loading, pressure, and many others. Equation (2) summarizes the factors that can contribute to the acoustic wave velocity shift:

$$
\frac{\Delta f}{\mathrm{fo}}=\frac{\Delta \mathrm{v}}{\text { vacoustic }}=\frac{1}{\mathrm{v}}\left(\frac{\mathrm{dv}}{\mathrm{dm}} \Delta \mathrm{m}+\frac{\mathrm{dv}}{\mathrm{d} \sigma} \Delta \sigma+\frac{\mathrm{dv}}{\mathrm{dc}} \Delta \mathrm{c}+\frac{\mathrm{dv}}{\mathrm{d} \varepsilon} \Delta \varepsilon+\frac{\mathrm{dv}}{\mathrm{dT}} \Delta \mathrm{T}+\frac{\mathrm{dv}}{\mathrm{dP}} \Delta \mathrm{P}+\frac{\mathrm{dv}}{\mathrm{d} \eta} \Delta \eta+\frac{\mathrm{dv}}{\mathrm{d} \rho} \Delta \rho+\ldots\right)
$$


where $\Delta \mathrm{m}$ is the change in mass load, $\Delta \sigma$ is the change in conductivity, $\Delta \mathrm{c}$ is the change in mechanical constant, $\Delta \varepsilon$ is the change in dielectric constant, $\Delta \mathrm{T}$ is the change in temperature, $\Delta \mathrm{P}$ is the change in pressure, $\Delta \eta$ is the change in viscosity and $\Delta \rho$ is the change in density.

To detect a change in the $\mathrm{H}_{3} \mathrm{O}^{+}$in a solution, a $\mathrm{pH}$-sensitive layer is necessary. In this work $\mathrm{ZnO}$ NPs have been used for this role. The significant difference in electronegativity between zinc (1.65) and oxygen (3.44) gives a sufficient negative charge on the latter to strip a proton from a neighboring $\mathrm{H}_{3} \mathrm{O}^{+}$[27] following Equation (3):

$$
\mathrm{ZnO}_{(\mathrm{s})}+\mathrm{H}_{3} \mathrm{O}^{+} \stackrel{\mathrm{Ka}}{\rightleftarrows} \mathrm{ZnOH}^{+}+\mathrm{H}_{2} \mathrm{O}
$$

where $\mathrm{Ka}$ is the dissociation constant of the equation.

The oxygen present in the atmosphere is absorbed onto the surface of the ZnO NPs layer as negatively charged ions capturing free electrons from the film. With the protonstripping reaction, surface oxygen ions are neutralized and the conductivity, $\sigma$, increases.

In the case of a layered structure Equation (2) becomes Equation (4):

$$
\frac{\Delta \mathrm{v}}{\text { vacoustic }}=-\mathrm{c}_{\mathrm{m}} \mathrm{f}_{0} \Delta \rho_{\mathrm{s}}+\mathrm{c}_{\mathrm{e}} \mathrm{f}_{0} \mathrm{~h} \Delta\left[\frac{\left(\frac{4 \mu}{\mathrm{v}_{0}^{2}}\right)(\eta+\mu)}{\eta+2 \mu}\right]-\left(\frac{\mathrm{K}^{2}}{2}\right) \Delta\left(\frac{\sigma^{2}}{\sigma^{2}+\mathrm{v}_{0}^{2} \mathrm{C}_{\mathrm{s}}^{2}}\right)
$$

where $f_{0}$ is the center frequency of the sensor, $\mathrm{cm}$ is the sensitive coefficient for the mass, $\rho_{\mathrm{s}}$ is the mass per unit area, ce is the sensitive coefficient for elasticity, $\mu$ and $\eta$ are the shear and bulk moduli of the film, $\sigma$ is the conductivity of ZnO NPs, and $\mathrm{K}^{2}$ and Cs the electromechanical coupling coefficient and the capacitance per length of the substrate material, respectively. All these terms contribute to the frequency shift.

Given that the mass and electric constant do not change during the interaction between the NPs and the solution, the variation in SAW velocity is influenced by a change in conductivity of the ZnO NPs layer, and Equation (4) assumes the form of Equation (5).

$$
\frac{\Delta \mathrm{v}}{\text { vacoustic }}=-\left(\frac{\mathrm{K}^{2}}{2}\right) \Delta\left(\frac{\sigma^{2}}{\sigma^{2}+\mathrm{v}_{0}^{2} \mathrm{C}_{\mathrm{s}}^{2}}\right)
$$

Hence, a conductive layer has been formed in the microchannel.

$\mathrm{ZnO}$ NPs are deposited inside a $\approx 300 \mu \mathrm{m}$ thick and $250 \mu \mathrm{m}$ large microchannel, exactly aligned between the two IDTs to avoid direct contact between the IDT and the liquid solution and the consequent damping of the wave. This variation in the wave velocity changes the resonance frequency of SAWs.

In this paper a novel hybrid PEN/AlN/SU-8 microfluidic sensor is presented. The fabrication and the characterization of a microfluidic channel directly on AlN/PEN flexible SAW devices are described. Through chemical-physical tests, the microchannel's wettability and, using mechanical tests, its conformability for wearable applications have been demonstrated. In particular, the microfluidic channel has been placed between the two interdigital transducers (IDTs) to let the analyzed fluids cross the $\mathrm{pH}$-sensitive $\mathrm{ZnO}-\mathrm{NPs}$ functionalized SAW-active region. The high-sensitivity of L-SAW on polymeric substrate for $\mathrm{pH}$ detection has been demonstrated.

\section{Materials and Methods}

\subsection{Sample Preparation}

AlN-based SAW devices were fabricated on $125 \mu \mathrm{m}$ thick polyethylene naphtalate (PEN), purchased from Teonex (code Q65HA) (Wilmington, DE, USA) with one pretreated surface, which improves the growth adhesion for material deposition. The PEN samples $\left(2.5 \times 2.5 \mathrm{~cm}^{2}\right)$ were fixed on silicon substrate, by a removable PDMS layer, in order to allow a uniform deposition and reduce the temperature stress that occurred during AlN multistep deposition. Hence, the overall $4.5 \mu \mathrm{m}$ thick AlN layer was grown on PEN samples through 
magnetron sputtering deposition (LAB18, Kurt J. Lesker Company, Jefferson Hills, PA, USA), achieving a film of $4.5 \mu \mathrm{m}$ thickness and a good effective piezoelectric coefficient (d33) ranging between 2.12 and $3.29 \mathrm{pm} / \mathrm{V}$ [13]. Samples were then washed in acetone, isopropanol and water, respectively, and then dried with $\mathrm{N}_{2}$ flow.

\subsection{Lithography Processes for Microfabrication of PEN/AlN/SU-8-Based Device}

In order to obtain a Lamb wave at $\approx 500 \mathrm{MHz}$, each PEN/AIN SAW device has been designed with two identical IDTs with the parameters reported in Table 1.

Table 1. Design parameters of the IDT electrode of SAW fabricated devices.

\begin{tabular}{cc}
\hline $\mathbf{N}^{\circ}$ Fingers & 40 Pairs \\
\hline Wavelenght $(\boldsymbol{\lambda})$ & $20 \mu \mathrm{m}$ \\
\hline Metallization Ratio & 0.5 \\
\hline Acoustic Aperture & $10 \lambda$ \\
\hline Distance between IDTs & $20 \lambda$ \\
\hline
\end{tabular}

The IDTs have been fabricated using bilayer photolithography carried out with Mask Aligner SUSS MA8/BA8 (Garching, Germany). LOR-10B (MicroChem), diluted 1:2 with $\mathrm{G}$ thinner (MicroChem, Westborough, MA, USA), was used as non-photosensitive resist followed by an AZ 5214 (MicroChemicals GmbH, Ulm, Germany) photosensitive layer [5]. Samples were washed in acetone, isopropanol and water, respectively, rinsed by $\mathrm{N}_{2}$ flow and treated with oxygen plasma (5'/150 W) (RFG300 Deiner, Ebhausen, Germany). Figure 1a shows the steps of the bilayer lithography process. After the photolithography, a $120 \mathrm{~nm}$ layer of aluminum was sputtered on the sample through magnetron sputtering deposition (LAB18, Kurt J. Lesker Company, Jefferson Hills, PA, USA). Usually, metal ion-free (MIF) developers are used for the lift-off process [28]. In our case the bilayer resistor was stripped out by Remover PG (MicroChem, Westborough, MA, USA), recommended for LOR-employed lithography. Figure $1 b-e$ shows the microscope images of the fabricated SAW device.

After bilayer photolithography, the sample is subjected to a photolithography process to pattern the microfluidic channel (Figure 1f), using the process described in Table 2 for SU82100 (MicroChem, Westborough, MA, USA), an epoxy-based negative photoresist, which allows us to obtain structures between 100 and $400 \mu \mathrm{m}$ thick. The microfluidics fabrication employs SU-8 photoresists, which are largely used for making microfluidics channels because of their optical transparency [29], good mechanical and chemical properties once polymerized [30,31] and biocompatibility [32], which are primary features for a biosensing wearable application. Figure $1 \mathrm{~g}$ shows the whole microfluidic aligned to the SAW device below. The optical images show an inlet diameter of $1500 \mu \mathrm{m}$, walls with a width of $50 \mu \mathrm{m}$ and a microchannel lumen of $250 \mu \mathrm{m}$ (Figure 1h). 


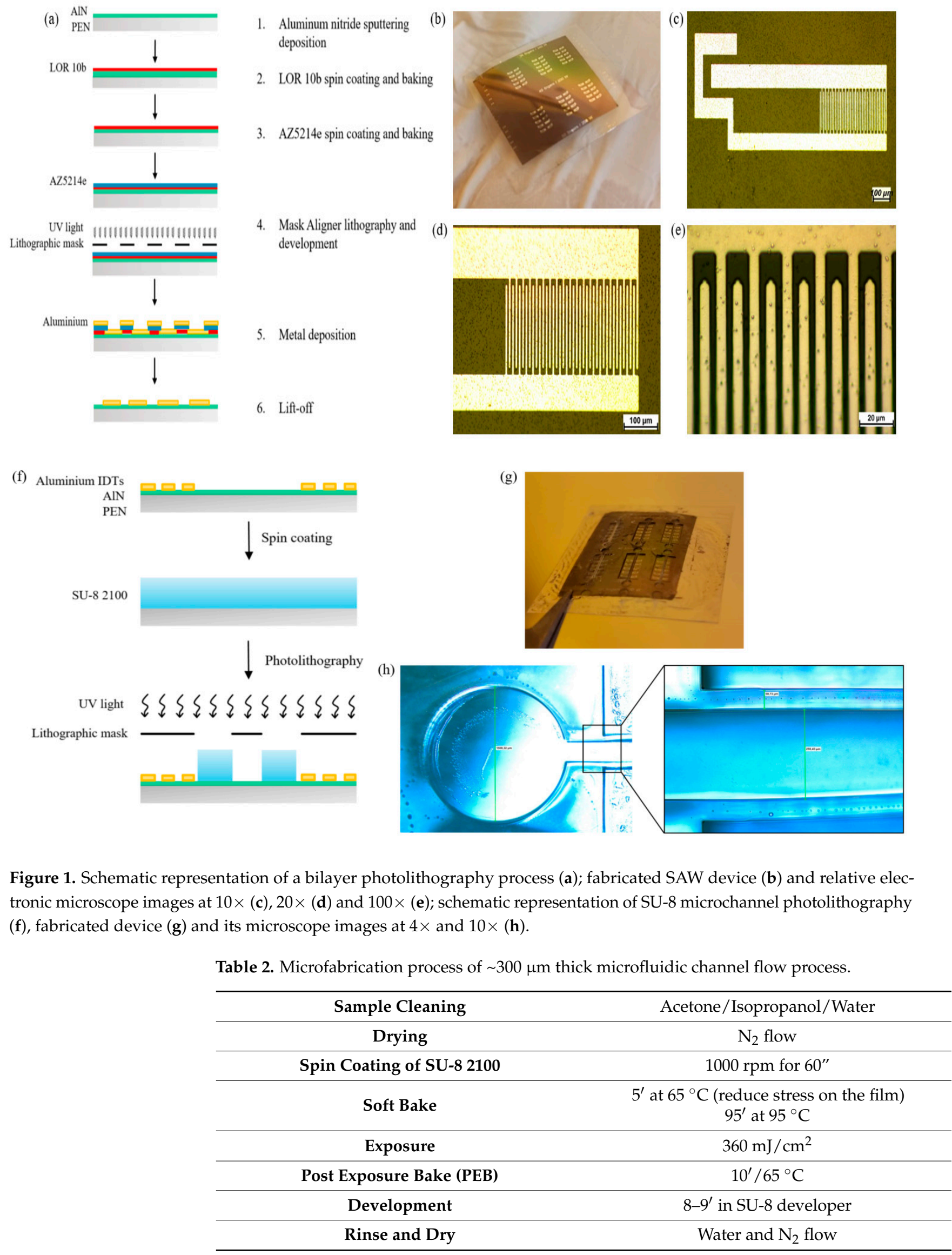




\subsection{ZnO Nanoparticles' (NPs) Synthesis and Microchannel Functionalization}

Zinc acetate dihydrate $\mathrm{Zn}\left(\mathrm{ac}_{2}\right) 2 \mathrm{H}_{2} \mathrm{O}$ and sodium hydroxide $(\mathrm{NaOH})$ have been used for the preparation of $\mathrm{ZnO}$ NPs. All the chemicals were purchased from Sigma Aldrich (St. Louis, MO, USA) and used without any further purification.

We have grown $\mathrm{ZnO}$ NPs following the protocol used by Adam et al. [33] starting from $\mathrm{Zn}\left(\mathrm{ac}_{2}\right) 2 \mathrm{H}_{2} \mathrm{O} 0.1 \mathrm{M}$ and $\mathrm{NaOH} 0.2 \mathrm{M}$ in deionized water. Then, we put together the two solutions into a becher and stirred at $500 \mathrm{rpm}$ for $2 \mathrm{~h}$ under a temperature of $60^{\circ} \mathrm{C}$. Then we applied centrifugation at $4500 \mathrm{rpm}$ for $2 \mathrm{~min}$ and the precipitate was washed with deionized water and acetone, respectively. Finally, the sample was dried in an oven at $75^{\circ} \mathrm{C}$ for $6 \mathrm{~h}$ and $\mathrm{ZnO}$ NPs in the form of powder were obtained.

Finally, microchannels were filled with a ZnO NPs solution of $50 \mathrm{mg} / \mathrm{ml}$ in ethanol by drop casting, and internal surfaces were functionalized by drying. NPs were deposited on the ground of the microchannel. The thickness of the deposited layer by drop casting was measured using a Dektak XT Profilometer (Billerica, MA, USA) showing a thickness of about $1.5 \mu \mathrm{m}$. This thickness was chosen because $\mathrm{pH}$ sensitivity is dependent on NPs layer thickness. Oh et al. have demonstrated a better linear response for a thickness around $1 \mu \mathrm{m}$ [2]. After these steps, the finished device (Figure 2) was tested.

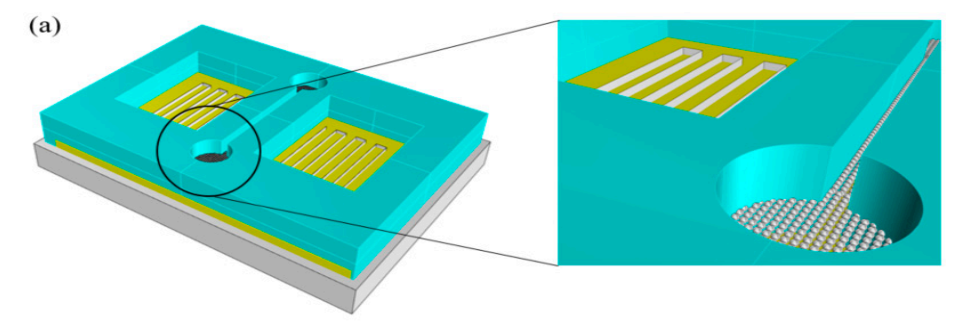

(b)

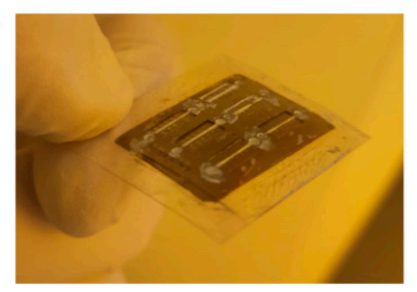

(c)

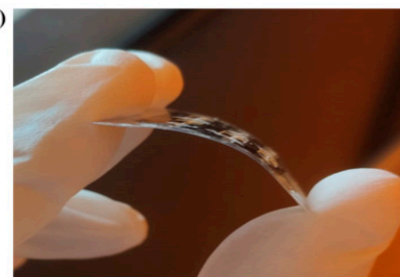

Figure 2. Tridimensional model of finished device (a); photo of the fabricated comformable PEN SAW-based device $(\mathbf{b}, \mathbf{c})$.

\subsection{Buffer Solutions Preparation}

All chemicals used to prepare buffer solutions for $\mathrm{pH}$ tests were purchased from Sigma Aldrich (St. Louis, MO, USA). In particular, $\mathrm{HCl} / \mathrm{KCl}$ buffer has been used to prepare $\mathrm{pH}$ $=2$ solution, sodium citrate dihydrate/citric acid buffers have been used for solutions from $\mathrm{pH}=3$ to $\mathrm{pH}=6$, and MilliQ water was used for $\mathrm{pH}=7$. All solutions' $\mathrm{pH}$ values have been adjusted to the final desired $\mathrm{pH}$ using $\mathrm{HCl}$ or $\mathrm{NaOH}$. A range of $\mathrm{pH}$ between 2 and 7 was chosen for this work in order to test the physiological value of $\mathrm{pH}$ in sweat.

\section{Results and Discussion}

\subsection{Mechanical Properties}

Mechanical characterization is fundamental to verify the conformability of a new hybrid material to human skin. Such devices should show the typical Young's modulus of the skin, which can be obtained through flexible substrates [34]. For this reason, the mechanical properties of the new PEN/SU-8 hybrid device ( $\mathrm{t}_{\text {PEN }} \sim 125 \mu \mathrm{m}$, $\mathrm{t}_{\mathrm{SU}}-8 \sim 300 \mu \mathrm{m}$ ) were assessed with a dynamic mechanical analyzer (DMA Q800, TA Instruments, USA). In particular, tensile strain was investigated (Figure 3).

Strip samples with dimensions of $40 \times 10 \mathrm{~mm}^{2}$ were used for tensile tests and a ramp force of $1 \mathrm{~N} / \mathrm{min}$ to $18 \mathrm{~N}$ at $25^{\circ} \mathrm{C}$ was used. In the linear-elastic region, Hooke's law can be 
applied to describe sample responses. The resulting Young modulus of the hybrid device was $0.92 \pm 0.04 \mathrm{GPa}$, comparable with the value of other flexible devices employed in skin-placed flexible biosensors [34].

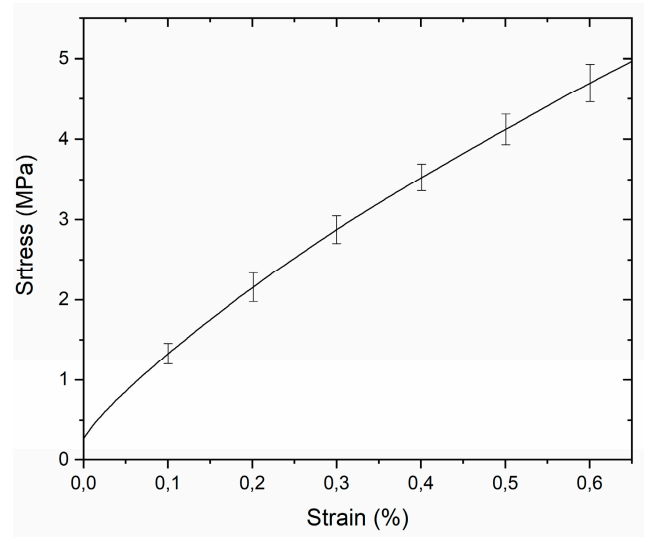

Figure 3. Resulting stress/strain curve in the linear-elastic range of the tensile strength of a PEN/SU-8 hybrid device.

\subsection{Chemical-Physical Properties of Microfluidic Functionalization}

The hydrophilicity of the channel is a key parameter in microfluidic SAW devices. The microfluidic channel must be highly hydrophilic to allow a water solution to pass through by use of capillary force [35]. For this reason, the contact angle measurements (undertaken with a Contact Angle System Oca 15 Pro (DataPhysics, Filderstadt, Germany) have been carried out using deionized water. PEN and SU-8 show contact angles of $80.92 \pm 2.73^{\circ}$ and $85.10 \pm 2.23^{\circ}$, respectively; these values show a weak hydrophilic character, unsuitable for microfluidics. In order to reduce the contact angle, an $\mathrm{O}_{2}$ plasma treatment has been performed on both substrates using an Oxygen Plasma Asher RFG 13.56/300 (Diener electronic, Ebhausen, Germany). An oxygen flow of $25 \mathrm{sccm}$ at a pressure of approximately $0.6 \mathrm{mbar}$ and at a power of 230 Watt was used for $5 \mathrm{~min}$. We performed contact angle goniometry to characterize the wetting behavior of the plasma-activated SU- 8 and PEN surfaces, and the measurements at $t_{0}$ show a huge decrease in the contact angle, which was $7.06 \pm 1.41^{\circ}$ for PEN and $18.19 \pm 1.88$ for SU-8 (Figure 4a,b). An aging test was conducted to analyze the efficacy through time of the treatment $[36,37]$. Aging measurements on PEN show a contact angle of $27.52 \pm 1.32^{\circ}$ after two weeks, with a negligible variation of about $20^{\circ}$ since $t_{0}$. On the other hand, SU-8 shows an angle of $73.71 \pm 1.82^{\circ}$ after two weeks, with a stabilization of the value after $96 \mathrm{~h}$ (Figure 4c); hence, even with SU-8 partially losing its hydrophilic character, it still has enough to allow water to go across it.

(a)

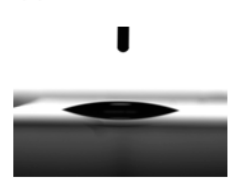

(b)

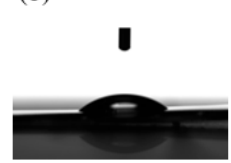

to
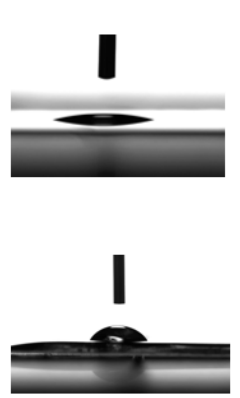

$24 \mathrm{~h}$
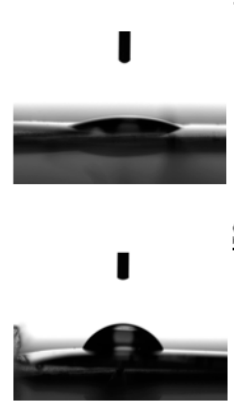

$48 \mathrm{~h}$
$\underline{\mathrm{PEN} \mathrm{O}_{2} \text { treated }}$
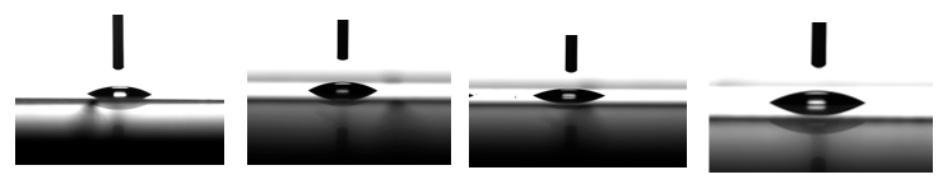

$\underline{\mathrm{SU}-8 \mathrm{O}_{2} \text { treated }}$
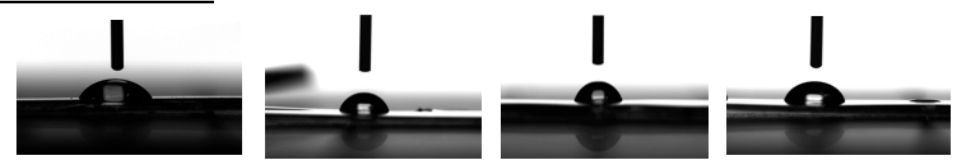

$72 \mathrm{~h}$
$96 \mathrm{~h}$ 1week

2weeks

Figure 4. Cont. 


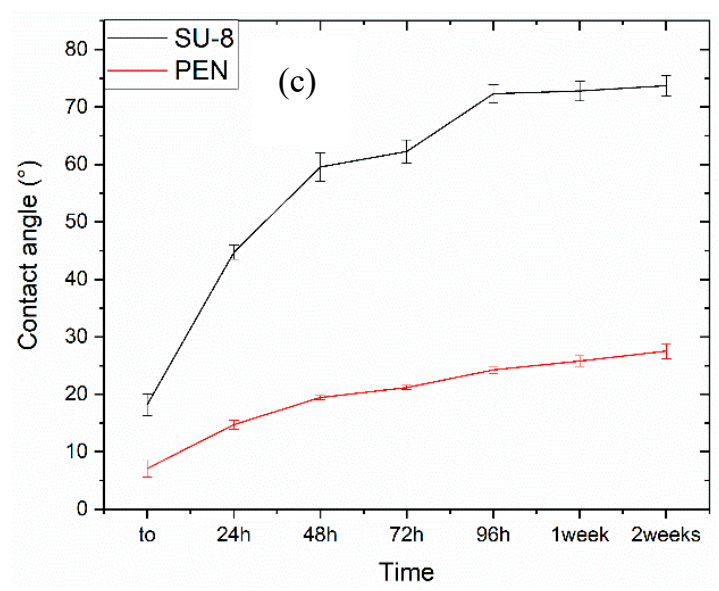

Figure 4. Images of PEN (a) and SU-8 (b) aging contact angle test and relative measurements at $\mathrm{t}_{0}, 24 \mathrm{~h}, 48 \mathrm{~h}, 72 \mathrm{~h}, 96 \mathrm{~h}$, one and two weeks (c).

\subsection{ZnO Nanoparticles (NPs) Characterization}

The ZnO NPs have been characterized using X-ray diffraction (XR) to evaluate the crystallographic quality of the sample using a Rigaku D-Max/Ultima (Tokyo, Japan) working at $40 \mathrm{kV}$ with a $\mathrm{Cu} \mathrm{K} \alpha$ radiation. In the XRD pattern (Figure 5), the resulting peaks correspond to the pure hexagonal wurtzite phase of $\mathrm{ZnO}$ [33]. These results allowed us to see that no other $\mathrm{ZnO}$ phases or impurities are present in the sample.

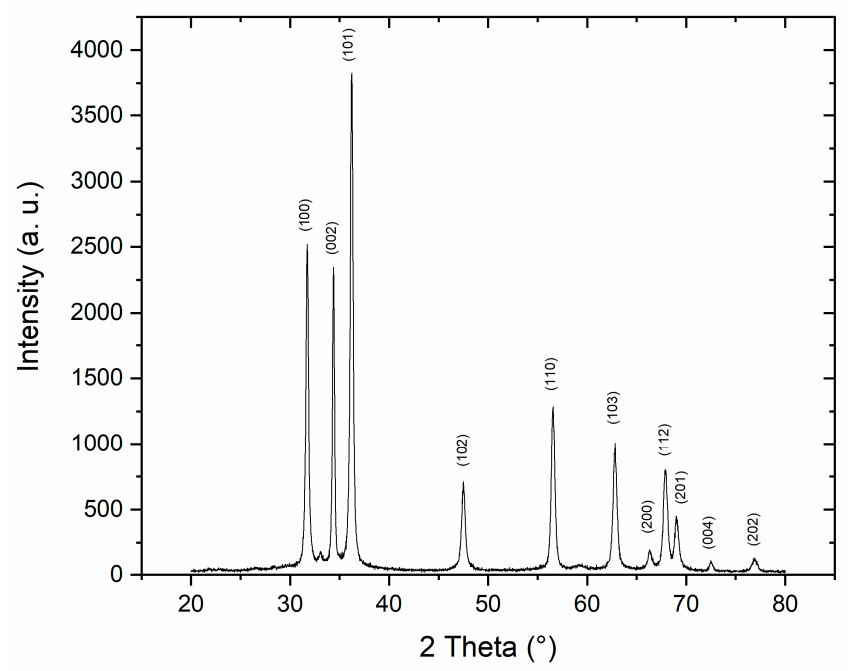

Figure 5. XRD spectrum of ZnO NPs.

The crystalline size was calculated using the Scherrer equation:

$$
\mathrm{D}=\frac{\mathrm{k} \lambda}{\beta \cos \theta}
$$

where $\mathrm{D}$ is the crystalline size of $\mathrm{ZnO}, \lambda$ is the $\mathrm{X}$-ray wavelength $(0.15406 \mathrm{~nm}), \theta$ is the Bragg diffraction angle, $\beta$ is the full-width at the half maximum (FWHM) of the diffraction peak corresponding to plane (101) and $\mathrm{k}$ is Scherrer constant (0.9). The resulting average crystalline size of $\mathrm{ZnO}$, calculated with this formula, is $25 \mathrm{~nm}$.

NPs dimensions, dependent on the speed of the addition of zinc acetate to $\mathrm{NaOH}$ solution during the synthesis [38], was evaluated using a dynamic light scattering (DLS) test through a Zetasizer Nano (ZS) (Malvern Panalytical, Malvern, UK)). The concentration of the samples was $1 \mathrm{mg} / \mathrm{mL}$ in a deionized water solution, and before the test they were 
sonicated for $90 \mathrm{~min}$. The results show NPs of $435.1 \pm 26.4 \mathrm{~nm}$ (Figure 6). Only one peak in the distribution was observed, demonstrating a uniform distribution of the dimensions.

$\begin{array}{rlllll} & & \text { Size (d.nm): } & \text { \% Intensity: } & \text { St Dev (d.nm): } \\ \text { Z-Average (d.nm): } & 435.1 & \text { Peak 1: } & 463.0 & 100.0 & 144.9 \\ \text { Pdl: } & 0.168 & \text { Peak 2: } & 0.000 & 0.0 & 0.000 \\ \text { Intercept: } & 0.888 & \text { Peak 3: } & 0.000 & 0.0 & 0.000\end{array}$

Result quality : Good

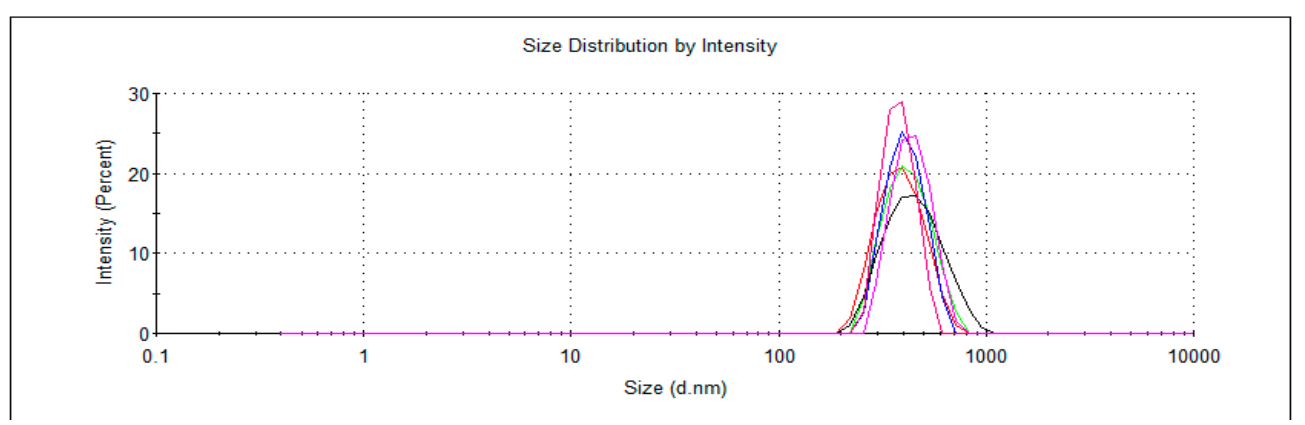

Figure 6. DLS analysis of ZnO NPs.

The morphology of the synthetized NPs has been evaluated by SEM analysis using a FEI Helios Nanolab 600i Dual Beam FIB/SEM system (Waltham, MA, USA). Before the SEM analysis, the samples were covered with a thin, $5 \mathrm{~nm}$ layer of gold with a sputter coater (Quorum, Q150 R S, Lewes, UK)) in order to improve the quality of the images. Via SEM, the shapes of the ZnO NPs have been characterized (Figure 7a,b). The NPs were uniform and showed a crystalline average size of $\approx 25 \mathrm{~nm}$ as calculated by the Scherrer equation. Furthermore, the dimensions of the ZnO NPs are coherent with the results of the DLS analysis, in which was found a dimension of about $430 \mathrm{~nm}$. Finally, high magnifications allowed us to see the hexagonal wurtzite shape (Figure 7c,d).

(a)

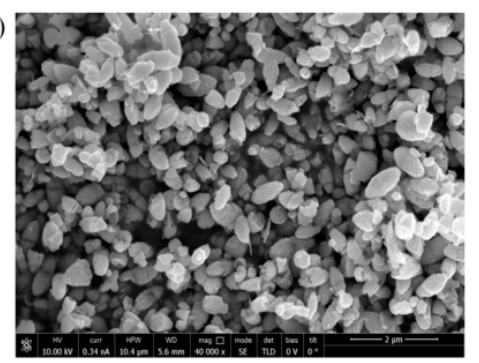

(c)

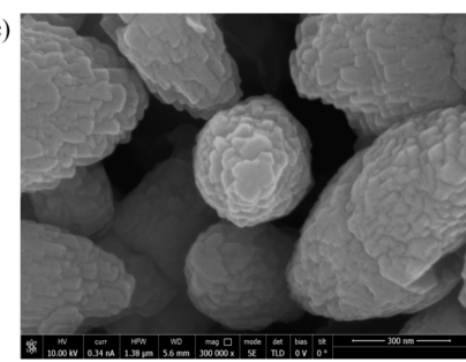

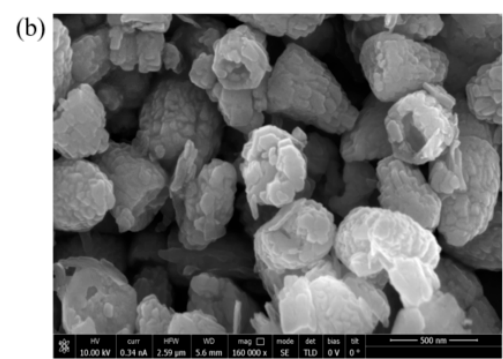

(d)

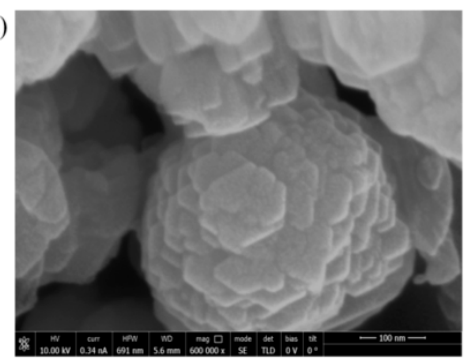

Figure 7. SEM images of the ZnO NPs at different magnifications; $40,000 \times(\mathbf{a}), 160,000 \times(\mathbf{b}), 300,000 \times$ (c) and $600,000 \times(\mathbf{d})$.

\subsection{Electroacoustic Response of the SAW Device}

The devices were connected to the VNA (Agilent 8753ES, Santa Clara, CA, USA) with a coplanar $|\mathrm{Z}|$ Probe (Electron $\mathrm{Mec}$ ) with a $150 \mu \mathrm{m}$ pitch and they were tested by measuring the transmission amplitude signal S21, obtained by a Vector Network Analyzer 
(Tektronix, TTR503A). The setup in Figure $8 \mathrm{a}$ was used to test the device at different $\mathrm{pH}$ values. All the devices have been tested in a controlled environment at $25^{\circ} \mathrm{C}$ and $30-40 \%$ of humidity. PEN devices show a clear resonance frequency corresponding to the propagation of Lamb SAW around $500 \mathrm{MHz}$. A transmission signal of about $15 \mathrm{~dB}$ is observed for these waves and a good phase response is clearly visible (Figure $8 b, c$ ). In this work all the devices were tested before the fabrication of the microchannel, after its fabrication and after the functionalization with NPs, to record the reference frequency. A significant frequency resonance decrease of about $375 \mathrm{kHz}$ was observed due to the SU-8 microchannel, because of its weight, but no significant decrease was observed after the functionalization with NPs due to their low weight. A significant frequency shift of $\approx 4 \mathrm{MHz}$ was observed when the first solution $(\mathrm{pH}=7)$ was added (Figure $8 \mathrm{~d})$.

(a)

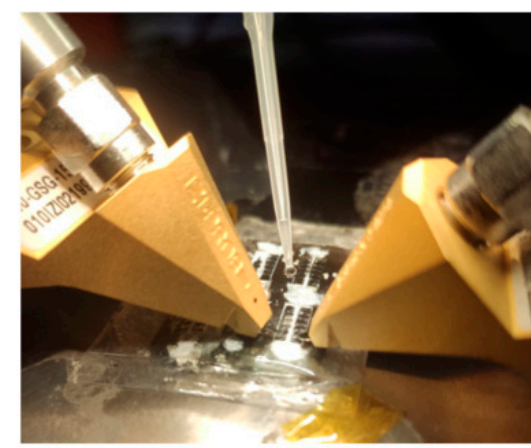

(c)

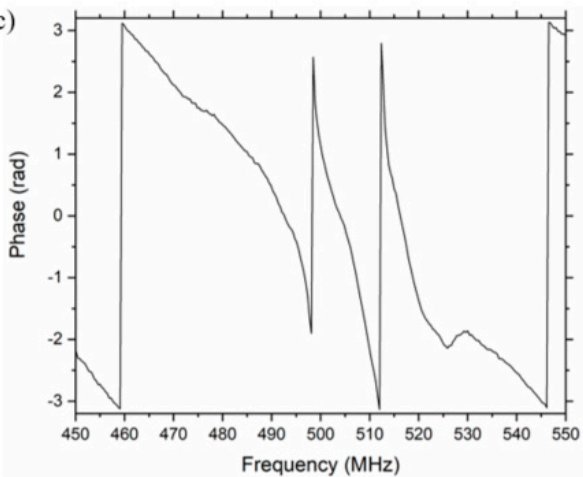

(b)
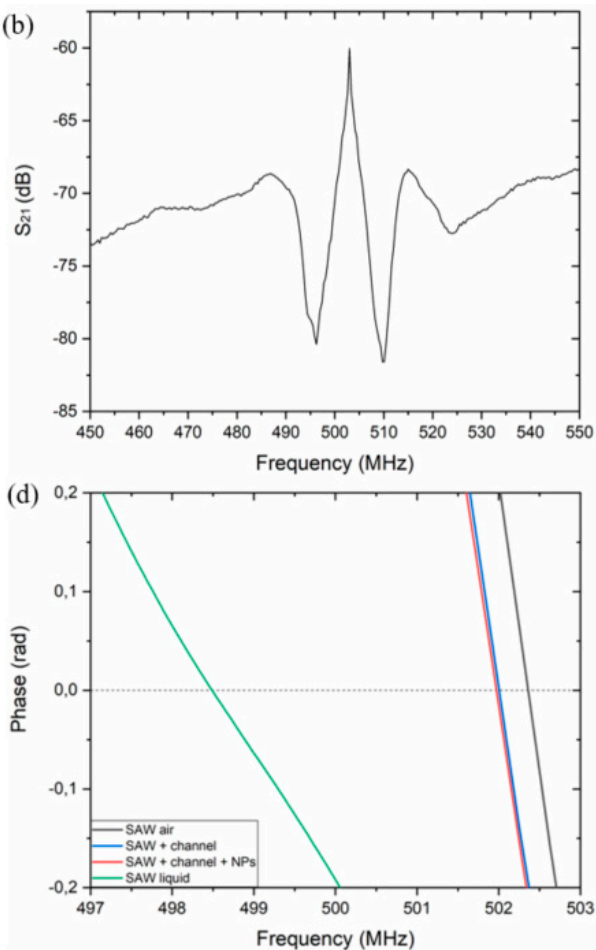

Figure 8. Experimental setup, used to study the electroacoustic response of the SAW devices (a), transfer function amplitude $S_{21}$ of the PEN device in air (b) and its phase response in air (c); changes in phase response of SAW device in air, after microchannel fabrication, after NPs deposition and the microchannel was filled with liquid (d).

Six solutions from $\mathrm{pH}=7$ to $\mathrm{pH}=2$, with a step of one unit, have been used to evaluate the electroacoustic response in the L-SAW resonance. The presence of a liquid medium induces a shift of about 3.7 MHz to the frequency of the device in air (Figure 8d). The resonance center frequency obtained with the $\mathrm{pH}=7$ solution has been used as a reference frequency, f0, in the experiment. In Figure 9a, the phase responses for PEN devices at different $\mathrm{pH}$ values are shown. The inset in Figure 9a clearly shows the center frequency shift for the different $\mathrm{pH}$ values. Figure $9 \mathrm{~b}$ reports the resonance shift $\left(\Delta \mathrm{f}=\mathrm{f}-\mathrm{f}_{0}\right)$, where $\mathrm{f}_{0}$ represents the center frequency at $\mathrm{pH}=7$ and $\mathrm{f}$ the center frequency of the other $\mathrm{pH}$ values. As expected, the center frequency of the sensor response decreases for lower $\mathrm{pH}$ levels due to the increase in ZnO NPs conductivity. The same device has been used to perform measurements at different $\mathrm{pH}$ values, after washing and drying the microchannel after each measurement, demonstrating that the shifts are reversible, and the device is reusable. In fact, according to Oh et al., no dissolution of $\mathrm{ZnO}$ NPs has been observed after exposure to a strong acid [2]. 

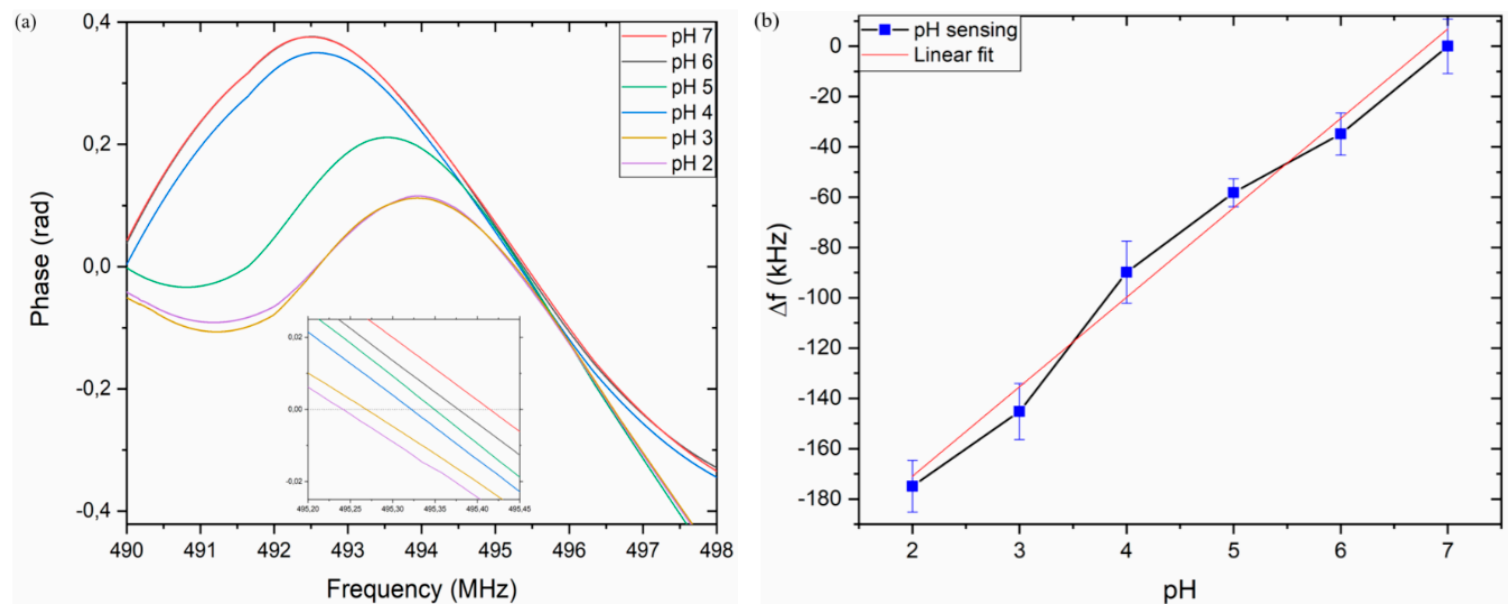

Figure 9. Phase change at different $\mathrm{pH}$ solutions, inset in figure shows a zoom of phase response (a); resonance frequency shift in response to different $\mathrm{pH}$ values (b).

A sensitivity of $\approx 30 \mathrm{kHz} / \mathrm{pH}$ has been observed; a linear response is obtained with a regression slope value with an $R^{2}=0.983$ and a total shift of $\approx 173 \mathrm{kHz}$ in the $\mathrm{pH}$ dynamic range between 2 and 7 .

\section{Conclusions}

A novel SU-8-based microfluidic, reusable, PEN/AIN SAW device based on ZnO NPs for $\mathrm{pH}$ sensing has been designed, fabricated, characterized and tested. The method consists of patterning a $\approx 300 \mu \mathrm{m}$ thick microfluidic channel between the two IDTs on a SAW device; then, the microchannel functionalization with ZnO NPs makes it sensitive to $\mathrm{pH}$, due to the $\mathrm{ZnO}$ NPs' changing conductivity with $\mathrm{pH}$, and the SAW Lamb resonance frequency of about $500 \mathrm{MHz}$. The microfluidic channel has shown a Young's modulus of $0.92 \pm 0.04 \mathrm{GPa}$, compliant with a wearable device, and a water contact angle of $27.52 \pm$ $1.32^{\circ}$ and $73.71 \pm 1.82^{\circ}$ for the PEN and SU-8 surfaces, respectively, demonstrating their hydrophilic character. The electroacoustic response has shown a sensitivity of $\approx 30 \mathrm{kHz} / \mathrm{pH}$ and a total shift of $\approx 173 \mathrm{kHz}$. To our best knowledge, this is the first time a microfluidic channel has been directly fabricated on a SAW flexible device for sensing applications. A specific functionalization of the microchannel could make it sensitive to specific biological parameters, such as cells mass loading, bacteria, viruses, and small molecules masses, paving the way to the miniaturization of the electroacoustic-based detection of biological samples in liquid.

Author Contributions: Conceptualization L.P., L.L., F.R. and M.D.V.; device fabrication, characterization and data curation L.P.; growth of piezoelectric substrate F.G.; SEM imaging A.B.; mechanical tests M.M.; writing—original draft L.P.; writing—review and editing L.P., L.L., F.R. and M.D.V. All authors have read and agreed to the published version of the manuscript.

Funding: This research received no external funding.

Acknowledgments: We would like to thank Donato Cannoletta for XRD analysis.

Conflicts of Interest: The authors declare no competing interests.

\section{References}

1. Fu, Y.; Luo, J.; Nguyen, N.; Walton, A.; Flewitt, A.; Zu, X.; Li, Y.; McHale, G.; Matthews, A.; Iborra, E.; et al. Advances in piezoelectric thin films for acoustic biosensors, acoustofluidics and lab-on-chip applications. Prog. Mater. Sci. 2017, 89, 31-91. [CrossRef]

2. Oh, H.; Lee, K.J.; Baek, J.; Yang, S.S.; Lee, K. Development of a high sensitive pH sensor based on shear horizontal surface acoustic wave with ZnO nanoparticles. Microelectron. Eng. 2013, 111, 154-159. [CrossRef]

3. Ramshani, Z.; Reddy, A.S.; Narakathu, B.B.; Wabeke, J.T.; Obare, S.O.; Atashbar, M.Z. SH-SAW sensor based microfluidic system for the detection of heavy metal compounds in liquid environments. Sens. Actuators B Chem. 2015, 217, 72-77. [CrossRef] 
4. Lamanna, L.; Rizzi, F.; De Vittorio, M.; Bhethanabotla, V.R. Light interaction with AlN-based SAW device fabricated on flexible and silicon substrate. In Proceedings of the IEEE Sensors, Montreal, QC, Canada, 27-30 October 2019; pp. 1-4.

5. Lamanna, L.; Rizzi, F.; Bhethanabotla, V.R.; De Vittorio, M. GHz AlN-based multiple mode SAW temperature sensor fabricated on PEN substrate. Sens. Actuators A Phys. 2020, 315, 112268. [CrossRef]

6. Lamanna, L.; Rizzi, F.; Bianco, M.; Agostini, M.; Cecchini, M.; De Vittorio, M.; Bhethanabotla, V.R. Photoresponse of the AlN-Based SAW Device on Polymeric and Silicon Substrates. IEEE Sens. J. 2021, 21, 9675-9681. [CrossRef]

7. Lamanna, L.; Rizzi, F.; Giudo, F.; De Vittorio, M. Flexible Dual-Wave Mode AlN-Based Surface Acoustic Wave Device on Polymeric Substrate. IEEE Electron Device Lett. 2020, 41, 1692-1695. [CrossRef]

8. Kidakova, A.; Boroznjak, R.; Reut, J.; Öpik, A.; Saarma, M.; Syritski, V. Molecularly imprinted polymer-based SAW sensor for label-free detection of cerebral dopamine neurotrophic factor protein. Sens. Actuators B Chem. 2020, 308, 127708. [CrossRef]

9. Jiang, Y.; Tan, C.Y.; Tan, S.Y.; Wong, M.S.F.; Chen, Y.F.; Zhang, L.; Yao, K.; Gan, S.K.E.; Verma, C.; Tan, Y.-J. SAW sensor for Influenza A virus detection enabled with efficient surface functionalization. Sens. Actuators B Chem. 2015, 209, 78-84. [CrossRef]

10. Di Pietrantonio, F.; Cannata', D.; Benetti, M.; Verona, E.; Varriale, A.; Staiano, M.; D'Auria, S. Detection of odorant molecules via surface acoustic wave biosensor array based on odorant-binding proteins. Biosens. Bioelectron. 2013, 41, 328-334. [CrossRef] [PubMed]

11. Kao, K.S.; Cheng, C.C.; Chen, Y.C.; Chen, C.H. The dispersion properties of surface acoustic wave devices on AlN/LiNbO3 film/substrate structure. Appl. Surf. Sci. 2004, 230, 334-339. [CrossRef]

12. Jin, H.; Zhou, J.; He, X.; Wang, W.; Guo, H.; Dong, S.; Wang, D.; Xu, Y.; Geng, J.; Luo, J.K.; et al. Flexible surface acoustic wave resonators built on disposable plastic film for electronics and lab-on-a-chip applications. Sci. Rep. 2013, 3, 2140. [CrossRef] [PubMed]

13. Lamanna, L.; Rizzi, F.; Guido, F.; Algieri, L.; Marras, S.; Mastronardi, V.M.; Qualtieri, A.; De Vittorio, M. Flexible and Transparent Aluminum-Nitride-Based Surface-Acoustic-Wave Device on Polymeric Polyethylene Naphthalate. Adv. Electron. Mater. 2019, 5, 1900095. [CrossRef]

14. Manjakkal, L.; Szwagierczak, D.; Dahiya, R. Metal oxides based electrochemical pH sensors: Current progress and future perspectives. Prog. Mater. Sci. 2020, 109, 100635. [CrossRef]

15. Lee, H.; Choi, T.K.; Lee, Y.B.; Cho, H.R.; Ghaffari, R.; Wang, L.; Choi, H.J.; Chung, T.D.; Lu, N.; Hyeon, T.; et al. A graphene-based electrochemical device with thermoresponsive microneedles for diabetes monitoring and therapy. Nat. Nanotechnol. 2016, 11, 566-572. [CrossRef]

16. Wencel, D.; Abel, T.; McDonagh, C. Optical Chemical pH Sensors. Anal. Chem. 2014, 86, 15-29. [CrossRef] [PubMed]

17. Wypych, G. PEN poly(ethylene 2,6-naphthalate). In Handbook of Polymers; Wypych, G., Ed.; Elsevier BV: Amsterdam, The Netherlands, 2012; pp. 372-376.

18. Bedia, E.L.; Murakami, S.; Kitade, T.; Kohjiya, S. Structural development and mechanical properties of polyethylene naphthalate/polyethylene terephthalate blends during uniaxial drawing. Polymer 2001, 42, 7299-7305. [CrossRef]

19. Meinschien, J.; Falk, F.; Hobert, H.; Stafast, H. Deposition of SiC and AlN thin films by laser ablation. Appl. Surf. Sci. 1999, 138-139, 543-548. [CrossRef]

20. Iriarte, G.F.; Rodríguez, J.G.; Calle, F. Synthesis of c-axis oriented AlN thin films on different substrates: A review. Mater. Res. Bull. 2010, 45, 1039-1045. [CrossRef]

21. Berg, N.G.; Paskova, T.; Ivanisevic, A. Tuning the biocompatibility of aluminum nitride. Mater. Lett. 2017, 189, 1-4. [CrossRef]

22. Basillais, A.; Boulmer-Leborgne, C.; Mathias, J.; Perrière, J. Influence of the growth conditions of AlN and GaN films by reactive laser ablation. Appl. Surf. Sci. 2002, 186, 416-422. [CrossRef]

23. You, Y.; Ito, A.; Tu, R.; Goto, T. Preparation of the c-axis oriented AlN film by laser chemical vapor deposition using a newly proposed $\mathrm{Al}(\mathrm{acac}) 3$ precursor. J. Cryst. Growth. 2013, 365, 1-5. [CrossRef]

24. Lamanna, L.; Rizzi, F.; Bhethanabotla, V.R.; De Vittorio, M. Conformable surface acoustic wave biosensor for E-coli fabricated on PEN plastic film. Biosens. Bioelectron. 2020, 163, 112164. [CrossRef] [PubMed]

25. Winkler, A.; Brünig, R.; Faust, C.; Weser, R.; Schmidt, H. Towards efficient surface acoustic wave (SAW)-based microfluidic actuators. Sensors Actuators A Phys. 2016, 247, 259-268. [CrossRef]

26. Kołodziejczak-Radzimska, A.; Jesionowski, T. Zinc Oxide-From Synthesis to Application. Rev. Mater. 2014, 7, $2833-2881$.

27. Al-Hilli, S.M.; Willander, M.; Öst, A.; Strålfors, P. ZnO nanorods as an intracellular sensor for pH measurements. J. Appl. Phys. 2007, 102, 084304. [CrossRef]

28. Ouattara, L.; Knutzen, M.; Keller, S.S.; Hansen, M.F.; Boisen, A. Double layer resist process scheme for metal lift-off with application in inductive heating of microstructures. Microelectron. Eng. 2010, 87, 1226-1228. [CrossRef]

29. Wang, X.; Chen, Y.; Banu, S.; Morgan, H.; Fu, S.; Cui, Z. High density patterns fabricated in SU-8 by UV curing nanoimprint. Microelectron. Eng. 2007, 84, 872-876. [CrossRef]

30. Abgrall, P.; Lattes, C.; Conédéra, V.; Dollat, X.; Colin, S.; Gué, A.M. A novel fabrication method of flexible and monolithic 3D microfluidic structures using lamination of SU-8 films. J. Micromech. Microeng. 2005, 16, 113-121. [CrossRef]

31. Blanco, F.J.; Agirregabiria, M.; Garcia, J.; Berganzo, J.; Tijero, M.; Arroyo, M.T.; Ruano, J.M.; Aramburu, I.; Mayora, K. Novel three-dimensional embedded SU-8 microchannels fabricated using a low temperature full wafer adhesive bonding. J. Micromech. Microeng. 2004, 14, 1047-1056. [CrossRef] 
32. Nemani, K.V.; Moodie, K.L.; Brennick, J.B.; Su, A.; Gimi, B. In vitro and in vivo evaluation of SU-8 biocompatibility. Mater. Sci. Eng. C. 2013, 33, 4453-4459. [CrossRef] [PubMed]

33. Adam, R.E.; Pozina, G.; Willander, M.; Nur, O. Synthesis of ZnO nanoparticles by co-precipitation method for solar driven photodegradation of Congo red dye at different $\mathrm{pH}$. Photon-Nanostruct.-Fundam. Appl. 2018, 32, 11-18. [CrossRef]

34. Choi, J.; Chen, S.; Deng, Y.; Xue, Y.; Reeder, J.T.; Franklin, D.; Oh, Y.S.; Model, J.B.; Aranyosi, A.J.; Lee, S.P.; et al. Skin-Interfaced Microfluidic Systems that Combine Hard and Soft Materials for Demanding Applications in Sweat Capture and Analysis. Adv. Health Mater. 2021, 10, 2000722. [CrossRef] [PubMed]

35. Olanrewaju, A.; Beaugrand, M.; Yafia, M.; Juncker, D. Capillary microfluidics in microchannels: From microfluidic networks to capillaric circuits. Lab Chip 2018, 18, 2323-2347. [CrossRef]

36. Walther, F.; Davydovskaya, P.; Zürcher, S.; Kaiser, M.; Herberg, H.; Gigler, A.M.; Stark, R.W. Stability of the hydrophilic behavior of oxygen plasma activated SU-8. J. Micromech. Microeng. 2007, 17, 524-531. [CrossRef]

37. Vesel, A.; Junkar, I.; Cvelbar, U.; Kovac, J.; Mozetic, M. Surface modification of polyester by oxygen- and nitrogen-plasma treatment. Surf. Interface Anal. 2008, 40, 1444-1453. [CrossRef]

38. Fernando, S.; Madusanka, N.; Kottegoda, N.; Ratnayake, U.N. Zinc Oxide Nanoparticles as an Activator for Natural Rubber Latex. In Proceedings of the International Conference on Advanced Materials, Science and Engineering, Colombo, Sri Lanka, 1-4 July 2012. 